\title{
Pour qui, pourquoi une ressource est-elle jugée dégradée? Pâturages et agriculture familiale au Brésil
}

\author{
Muriel Figuié ${ }^{1}$, Bernard Hubert ${ }^{2}$ \\ 1 Sociologue, CIRAD, UMR MOISA, 34398 Montpellier cedex 5, France \\ 2 Écologue, INRA, SED, 75338 Paris cedex 7, France
}

Cet article remet en question le principe d'autorité dont bénéficient des techniciens agricoles. La légitimité que leur confèrent les références scientifiques dont ils se recommandent dans leurs prescriptions en est le fondement. En retour, un tel principe disqualifie les connaissances des éleveurs sur la conception et la gestion des ressources prairiales. Les auteurs, partant d'une recherche de terrain qui montre le dépassement de cet antagonisme initial entre techniciens et éleveurs, proposent une modélisation de l'interface pratiques/représentations-connaissances/ressources et un concept permettant d'en renouveler l'approche.

La Rédaction

\section{Mots-clés :}

agriculture familiale ; Brésil ; dégradation ; pâturage ; représentation sociale

\section{Keywords:}

Brazil; degradation; pasture; small-scale farming; social representation
Résumé - Les concepts de ressource naturelle et de dégradation sont des constructions sociales. En témoignent les points de vue confrontés ici des transformations connues par la région brésilienne des Cerrados depuis les années 1960, assimilées à une mise en valeur par les uns et à un appauvrissement, voire une agonie, par les autres. La confrontation des points de vue des éleveurs (de type familial), à propos des pâturages et de leur dégradation, à ceux des techniciens confirme encore ce statut de construction sociale. Elle montre aussi que l'adoption matérielle d'innovations techniques (ici la fertilisation et la gestion tournante des pâturages) par les éleveurs nécessite également de leur part une reconstruction, voire une construction, des concepts de ressource et de dégradation. Cela témoigne plus généralement d'une activité conceptuelle socialement partagée et non confinée aux seuls lieux de la production officielle de connaissances. Cette analyse invite pour conclure à penser la gestion durable des ressources naturelles en termes d'intégrité fonctionnelle plutôt qu'en termes de stocks.

\begin{abstract}
The concept of degradation of a natural resource, a social construct. Pastures and small-scale farms in Brazil. The concepts of "natural resource" and "degradation" are social constructs. This point is emphasized by analyzing the discourses of Brazilian NGOs and agricultural research institutes working in the region of Cerrados in Central Brazil: their diagnoses on the colonization process of the region (from the 60 's on) range from an "area of impoverishment" for the NGOs, to a "New Eldorado" for agricultural research institutes. These divergences highlight ideological and interest conflicts about what should be the "good uses" and "good users" of this region. They are also evident when analyzing the question of pasture degradation in small-scale farms in the Silvânia commune in the State of Goiás. The recent increase of dairy production in the small-scale farms of this commune has been sustained partly by an intensified use of pastures (e.g. strip grazing) as part of a wider technological package. The concept of pasture degradation was introduced with this innovation; it is mainly assessed in terms of the technical production potential and mainly concerns the soil. This concept has replaced the concept of "weakening", which concerns mainly the plant cover and is assessed by comparing it with the native vegetation. It has been shown that the adoption of the degradation concept by farmers results from a change in the function of their pasture, rather than from a change in its state. It also becomes integrated into different representations of the animal farming system, which have shifted from a set of interactions to a representation in term of resource stocks. This analysis invites us to view the concept of sustainable development in terms of functional integrity of resources rather than in terms of managing resource stocks.
\end{abstract}

Auteur correspondant : muriel.figuie@cirad.fr 


\section{Introduction}

De nombreuses études soulignent l'impact de l'activité agricole sur la dégradation des ressources naturelles. Peu nombreuses, cependant, sont celles qui analysent le comportement des agriculteurs face à un problème qui menace les bases mêmes de leur activité. Ces derniers sont généralement supposés passifs - ou au pire conservateurs, voire obscurantistes - soit par inconscience, soit par impuissance. Cet article $^{1}$ a pour premier objectif de dépasser de telles positions pour étudier comment les agriculteurs abordent la question de la dégradation des ressources naturelles nécessaires à leur activité et comment cela peut aider à concevoir d'autres cadres théoriques d'approche de la question des ressources naturelles. Ce faisant, nous sommes amenés à confronter le point de vue de ces agriculteurs à ceux des techniciens et des experts, lesquels sont supposés détenir la légitimitéde qualifier les ressources de dégradées. Cette confrontation nous sert de base à une réflexion sur le statut de ressource naturelle et le jugement de dégradation. Notre objectif est de montrer que le concept de ressource naturelle, et en conséquence celui de dégradation, sont des constructions sociales : l'accès d'un élément du milieu naturel au statut de ressource dépend de l'appareillage technique, politique et moral de la société qui le reconnaît comme tel. Nous nous situons ainsi dans une nouvelle approche de la gestion des ressources naturelles, telle que définie par Hubert et Ison (2011), fondée sur une vision dynamique et interactive de ce qui fait ressource pour un système de production.

Les fronts pionniers agricoles se prêtent particulièrement bien à cette démonstration. En effet, dans une dynamique de colonisation, se définissent les fonctions des espaces, dans un environnement sociopolitique souvent tendu. Le terrain d'étude choisi est la région des Cerrados au Brésil et, dans l'État du Goiás, la commune de Silvânia en particulier. Cette région centrale du Brésil, dont la phase la plus intense de la colonisation date des années 1960, nous offre le recul nécessaire à l'analyse du processus de «mise en ressources» d'un territoire. En outre, ce processus fait l'objet d'une controverse : s'y affrontent des discours opposés sur l'évaluation de la gestion qui est faite des ressources naturelles par les agriculteurs.

Vu l'intensité des débats, il est difficile d'ignorer que les agriculteurs, au-delà de la gestion d'une seule réalité

1 Il est en majeure partie issu du travail de thèse de M. Figuié (2001), INA P-G, Paris. Cette thèse a été menée dans le cadre du projet Silvânia (1985-1998), projet de recherche-développement franco-brésilien coordonné par le Centre de coopération internationale en recherche agronomique pour le développement (Cirad) et l'Empresa Brasileira de Pesquisa Agropecuária (EMBRAPA). matérielle, ont à gérer un ensemble d'informations, de messages techniques et de connaissances qui leur proviennent des établissements de recherche, des organismes de développement, mais aussi des ONG qui mènent également des recherches et portent des diagnostics sur les questions d'environnement dans cette région.

Cette étude s'intéresse à la construction sociale de savoirs, tant au niveau de ce que Darré (1985) appelle les lieux officiels de production de connaissances qu'au niveau du lieu de l'action technique - ou celui des praticiens, c'est-à-dire ici les éleveurs de la commune de Silvânia.

\section{Pratiques et représentations}

De nombreux auteurs soulignent qu'il serait caricatural d'opposer deux niveaux, celui de la connaissance et celui de l'action, en posant que l'objectivisme et l'activité de conception caractériseraient le premier, le subjectivisme et l'activité de perception, le second. Les travaux de Latour (1995) et de Callon et Rip (1992) montrent que ce qui est produit par les milieux de la recherche est marqué par le contexte de cette production et plus précisément par les rapports de force au sein de la société. Cette conception relativiste de la connaissance scientifique, ce refus de "l'autoritarisme du vrai», ne doivent cependant pas céder la place à un « sociocentrisme » qui négligerait la réalité matérielle des problèmes abordés (Larrère et Larrère, 1997). Par ailleurs, les travaux de Darré (1999) réfutent la vision d'une société divisée entre concepteurs et exécutants. Le lieu de l'action est aussi un lieu où s'élaborent des savoirs. En particulier, l'adoption d'un changement technique se double d'une activité conceptuelle collective qui vise à donner un sens aux éléments de ce changement et à les intégrer au système conceptuel existant. Enfin, les travaux de Hatchuel (2005) sur les régimes de conception éclairent ces couplages entre logiques d'action et logiques de production de connaissances quand des régimes multiples et ouverts traversent des collectifs et nous permettent d'en rendre compte en tant que processus à la fois social et cognitif.

Pour étudier ces différentes formes de production de savoirs, nous avons fait appel au concept de représentations sociales, dont on a retenu l'aspect dynamique (Moscovici, 1961), considérant ces représentations comme des processus et des matrices de perception, d'appréciation et d'action (Bourdieu et Wacquant, 1992). Le modèle théorique construit à partir des contributions de ces différents auteurs (Darré en particulier) est représenté par la figure 1. Il cherche à traduire :

- qu'il existe une relation dialectique entre pratique et représentation, et non pas une relation de subordination de l'une à l'autre (flèche 1$)$; 
Contexte historique, politique, économique, social, institutionnel et biophysique.

lieu officiel de la connaissance

\author{
lieu de l'action technique
}

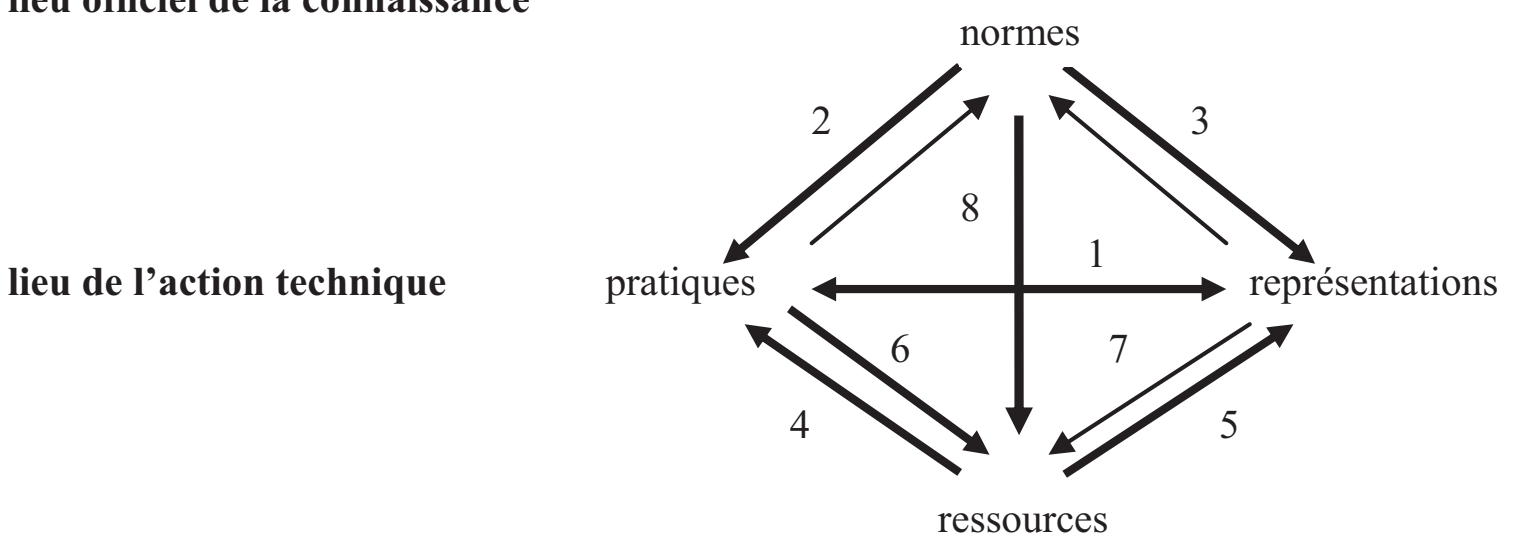

Fig. 1. Le modèle théorique adopté.

- que ce que produisent chercheurs et experts (les porteurs de la connaissance officielle) s'impose en tant que normes aux praticiens (ici les éleveurs) [flèches 2 et 3]. En revanche, les connaissances produites par le monde des praticiens ne pénètrent que très peu le lieu de la connaissance officielle. Cette dissymétrie est avant tout liée non pas tant aux valeurs des connaissances produites qu'à la différence de position sociale occupée par ceux qui les produisent ;

- que le couple pratiques-représentations est le produit à la fois des structures sociales qui s'expriment dans le pouvoir d'imposition des normes (flèches 2 et 3 ) et du lien des praticiens à une réalité matérielle (flèches 4 et 5). Ces deux versants sont en réalité indissociables dans leur déterminisme des pratiques et des représentations ;

- qu'au niveau du lieu de l'action, les normes peuvent être adoptées sous deux formes: dans les pratiques (adoption matérielle, flèche 2) et dans les représentations (adoption idéelle, flèche 3) ;

- que ces pratiques ont, in fine, un impact sur les ressources elles-mêmes (flèche 6) ;

- que le statut de ressource naturelle n'est pas lié aux caractéristiques intrinsèques de la ressource elle-même. Un élément du milieu naturel devient ressource pour un groupe social à partir du moment où celui-ci dispose de l'appareillage technique (technologie, organisation..., flèche 6) et du système de pensée (connaissance, morale..., flèche 7) pour l'exploiter, ainsi que du pouvoir politique nécessaire pour légitimer cet usage (flèche 8 ).

En nous intéressant aux acteurs du lieu de l'action, nous les considérons comme les acteurs d'un système sociotechnique plus vaste, à la manière des approches en termes de «multi-level perspective on system innovations » (Geels, 2004). L'importance relative des différentes flèches est ainsi liée à un contexte - un paysage, pour reprendre la terminologie de Geels (ibid.) - économique, politique, institutionnel, social et biophysique, car nous sommes bien en présence de systèmes non stabilisés, traversés par une diversité de régimes de conception, euxmêmes ancrés dans des logiques sociales et économiques dynamiques et en partie contradictoires. Si chaque élément du système a une relative autonomie dans la production de connaissances, liée à des perceptions, une histoire, un agenda qui lui est propre, il existe aussi une diversité au sein de chacun des éléments, que nous étudierons sur le lieu de l'action. À travers le suivi d'une innovation technologique, nous proposons d'analyser au niveau des "usagers des technologies »-trop souvent négligés des études sur les innovations sectorielles (ibid.) - la relative autonomie du travail de construction de sens par ce groupe, mais aussi les interactions avec d'autres acteurs du système sociotechnique, ici les techniciens. C'est l'analyse de l'ensemble de ce travail et de ces interactions qui nous permet de comprendre l'évolution du rapport des éleveurs aux ressources naturelles par le biais de l'innovation technologique. Ce rapport s'inscrit dans une histoire où, du côté des lieux légitimes de production de connaissances, le paradigme dominant est celui de l'équilibre définissant une capacité de charge, paradigme de plus en plus remis en question au profit d'approches par les systèmes complexes, incertains et non équilibrés (Scoones et al., 2007). Le modèle d'analyse proposé justifie la large place accordée dans notre travail à l'étude des pratiques des éleveurs. 
Par ailleurs, nous basant toujours sur les travaux de Darré, nous avons considéré qu'il était possible d'étudier les représentations d'un groupe à travers le sens que les individus qui le constituent donnent aux mots, chaque groupe étant un lieu de langage partagé. Nous avons donc fait de l'analyse de discours un instrument privilégié.

\section{Ressources et dégradation : des constructions sociales}

Si les transformations de l'environnement sont des faits que l'on peut décrire objectivement, qualifier ces transformations de positives ou de négatives (c'est-à-dire, dans ce dernier cas, parler de dégradation) ne peut se faire que par rapport à un objectif, une fonction attribuée aux ressources considérées. Or, l'attribution de cette fonction ne va pas de soi.

Le cas des Cerrados permet d'illustrer ce propos. L'unité et le nom de la région lui sont donnés par sa végétation naturelle, une savane arbustive. Depuis les premières incursions portugaises et espagnoles au $\mathrm{XVI}^{\mathrm{e}}$ siècle (dans une région à l'origine très faiblement peuplée par des populations indiennes que l'on connaît peu et qui furent massacrées), les Cerrados sont passés par diverses représentations en termes de ressources: réserves de terres attribuables par la Couronne portugaise en rétribution des chefs des corps expéditionnaires, réserves aurifères, puis (à l'épuisement de ces gisements d'or au $\mathrm{XIX}^{\mathrm{e}}$ siècle) réserves de pâturages naturels pour de grands élevages extensifs qui marquent le début de l'histoire agraire de la région (Bertran, 1988 et 1994). La représentation dominante des Cerrados en termes de ressources est donc évolutive. Nous nous concentrons ici sur la période suivante, celle qui démarre dans les années 1960, pour détailler, au-delà des représentations dominantes, la diversité de ces représentations, des logiques, des valeurs et des intérêts des acteurs qui les portent.

Les politiques de colonisation et de modernisation agricole qui ont été menées dans la région des Cerrados depuis les années 1960 ont conduit à une réduction des surfaces de végétation naturelle au profit de l'installation de grandes exploitations se consacrant soit à l'élevage bovin extensif sur des pâturages en grande partie artificiels, soit à la culture du soja (Pinto, 1993). Ces transformations sont considérées comme :

- une mise en valeur par les institutions en charge du développement et de la recherche agricole, qui voient les Cerrados comme le grenier du Brésil ${ }^{2}$ ou un nouvel Eldorado $^{3}$;

- un appauvrissement par les mouvements associatifs agissant dans le secteur social et environnemental, qui

2 Revista dos Criadores, 03/1997.

3 Globo Rural, 1998. dénoncent : « Les Cerrados agonisent dans l'indifférence générale », ou encore : " Pour les Cerrados la seule perspective est celle du désert et pour le peuple celle de la misère » (INESC, 1994).

Ces différents acteurs regardent bien le même espace : les Cerrados, mais ils n'en retiennent pas les mêmes éléments et ne les évaluent pas de la même manière.

Pour les agronomes de la recherche et du développement, le statut de « ressource » accordée à la région tient à la disponibilité d'espaces non encore cultivés. Les Cerrados sont un espace vierge, de faible potentiel agronomique du fait essentiellement de l'acidité naturelle des sols. Mais c'est aussi un espace à conquérir : les instruments de cette conquête sont la technologie, laquelle a permis en particulier d'améliorer la fertilité des sols et de construire ainsi le potentiel de la région. « La transformation des Cerrados en sols fertiles est une grande réalisation des Brésiliens [...]. Il est totalement absurde, pour des raisons environnementales, de vouloir en limiter l'exploitation alors que c'est cette exploitation qui corrige ce que la nature a dégradé », affirme un chercheur agronome brésilien ${ }^{4}$.

Pour les ONG sociales et environnementales, la région des Cerrados, loin de constituer un espace vierge, se caractérise par sa diversité biologique (cette région est parfois considérée comme une des savanes les plus riches du monde du point de vue génétique [Pinto, 1993]) et culturelle (notamment associée aux peuplements indiens et aux savoirs traditionnels des petits producteurs et des travailleurs ruraux), qui en fondent le statut de ressource (Duarte et al., 1998).

Ces différences traduisent ainsi un conflit social et économique sur la fonction à attribuer à la région. L'intégration de la région à l'économie nationale et internationale, à travers sa colonisation et sa modernisation agricole, est depuis les années 1960 l'objectif prioritaire des pouvoirs publics. Ces derniers considèrent que les ressources de la région doivent être confiées à ceux les plus à même de les exploiter, notamment par la production de soja ou l'élevage bovin sur des pâturages cultivés. Ce projet justifie, selon un ancien directeur du centre de recherches agronomiques consacré à la région des Cerrados (EMBRAPACerrados) \{Macedo, 1995], « l'élimination des producteurs les moins efficients » au profit des producteurs qui disposent déjà d'un capital (économique, éducatif) pour absorber les technologies modernes et de surfaces suffisantes pour valoriser les investissements ( 300 hectares au minimum). Ce projet justifie donc l'élimination des petits producteurs au profit des plus grands, qui en sont considérés comme les «bons usagers »... Les Cerrados sont alors qualifiés de «Terre des Géants ${ }^{5}$ ».

\footnotetext{
4 Gazetta Mercantil, 09/02/1998.

5 Veja, 1994.
} 
Dans les Cerrados, les ONG sont pour la plupart, à l'origine, des ONG sociales ayant intégré plus récemment un discours environnementaliste (ce qui est probablement différent des ONG qui interviennent depuis plus longtemps et en beaucoup plus grand nombre dans la région amazonienne). Leur objectif affiché est la recherche d'une plus grande équité sociale et la préservation de l'environnement ; elles abordent en conséquence les Cerrados comme un espace à la fois à partager et à préserver. La nécessité d'appuyer l'agriculture familiale, et la réforme agraire, constitue alors un impératif social et moral. Mais c'est aussi un impératif qualifié de technicoenvironnemental. En particulier parce que, comme l'écrivent les ONG, «le fonctionnement économique de l'exploitation familiale n'est pas fondé sur l'obtention de gains à court terme, mais sur la satisfaction des besoins familiaux et la préservation à long terme du potentiel productif du milieu naturel perçu comme un patrimoine familial » (Forum brasileiro de ONGs e movimentos sociais, 1997, p. 92). Les exploitations familiales représentent donc pour elles les « bons usagers».

Ces deux types de discours, totalement opposés, sont à l'image de la forte dualité de l'agriculture brésilienne. Cependant, entre ces extrêmes, il existe un continuum de points de vue plus nuancés, mais qui peinent à se faire entendre.

On constate ainsi que les discours sur la dégradation sontl'occasion de renforcer des alliances entre les groupes sociaux. Ils permettent également d'affirmer ou réaffirmer diverses attitudes vis-à-vis du progrès technique et des relations hommes-nature. Le discours sur la dégradation a donc indéniablement une dimension idéologique et politique. Et c'est cette dimension qui lui donne son sens, tout autant que l'état des ressources naturelles. S'interroger sur la dégradation exige d'analyser cette construction. Pour mener à bien cette analyse, nous nous sommes intéressés à une ressource en particulier: les pâturages, sur lesquels il était plus aisé de formaliser les raisonnements, et de procéder :

- à l'étude des diagnostics portés sur les pâturages et l'élevage (appréhendés par l'analyse de rapports techniques et $\mathrm{d}^{\prime}$ articles scientifiques et par une douzaine d'entretiens) par les chercheurs et les techniciens impliqués dans les organismes publics de développement de la région (EMBRAPA-Cerrados, EMATER ${ }^{6}$ ); ils sont pour nous les représentants du lieu de production officielle de connaissances. Certes, les techniciens ont un rôle délicat de médiateurs entre ce milieu scientifique, dont ils doivent diffuser les recommandations de type «one-fits-all», et les agriculteurs, dont ils connaissent

\footnotetext{
6 L'EMBRAPA (Empresa Brasileira de Pesquisa Agropecuária) et l'EMATER (Empresa de Assistência Técnica e Extensao Rural) sont deux instituts publics, l'un de recherche agronomique, l'autre de vulgarisation agricole.
}

probablement toute la diversité et la complexité des prises de décision, étant parfois eux-mêmes éleveurs. Cependant, lors de nos entretiens, ils se sont faits clairement porteurs du discours des scientifiques (les situations d'interactions avec les éleveurs auraient sans doute mérité d'être davantage analysées pour explorer ce rôle de médiateur, mais tel n'était pas notre propos) ;

- à des entretiens (quarante-trois, menés entre 1997 et 1999) avec des éleveurs familiaux de la commune de Silvânia (ceux-ci ont été sélectionnés avec l'aide des techniciens agricoles de la commune afin de couvrir la diversité des systèmes fourragers de la zone). Les entretiens ont porté sur la façon dont ces éleveurs définissent les pâturages, les classent, les insèrent dans un système de production et enfin les évaluent. Les entretiens menés avec vingt de ces éleveurs (au cours de plusieurs rencontres avec chacun) ont donné lieu à une analyse de discours selon la méthode mise au point par Darré (1985). Ces éleveurs sont pour nous les représentants du lieu de l'action technique.

\section{La dégradation des pâturages : le discours performatif scientifique}

La quantification du phénomène de dégradation des pâturages dans la région des Cerrados repose principalement sur deux types d'indicateurs, que l'on peut qualifier d'indicateurs de moyens :

- le pourcentage des surfaces de pâturages conduites selon les recommandations élaborées par les chercheurs de l'EMBRAPA-Cerrados et diffusées par les techniciens. Ainsi, constatant, sur la base d'une enquête auprès d'éleveurs, que sur 50 à $80 \%$ des surfaces les éleveurs n'ont pas ressemé, corrigé ou fertilisé leurs pâturages dans les délais ou selon les doses recommandées par les techniciens, l'EMBRAPA-Cerrados évalue que 50 à $80 \%$ des pâturages de la région sont dégradés (Barcellos, 1996) ${ }^{7}$. $\mathrm{C}^{\prime}$ est donc à partir de l'écart entre les recommandations des techniciens et les pratiques des éleveurs qu'est jugée ici la dégradation des pâturages ;

- la charge animale $\left(\mathrm{UA} / \mathrm{ha} / \mathrm{an}^{8}\right)$. On constate à partir de1985, sur la base des statistiques produites par l'Institut

\footnotetext{
7 Alors que dans le même temps les ONG, se basant sur le fait que $37 \%$ des surfaces des Cerrados sont anthropisées (sont comptabilisées les surfaces où la végétation naturelle a été remplacée par des pâturages artificiels ou des cultures), concluent : « $37 \%$ des surfaces des Cerrados sont dégradées » (Duarte et al., 1998).

8 Une unité animale (UA) correspond à une vache adulte tarie de $445 \mathrm{~kg}$ qui consomme $12 \mathrm{~kg}$ de matières sèches par jour. Cette unité est utilisée notamment pour le calcul de la charge globale des pâturages (UA/ha/an, nombre moyen d'UA divisé par la surface exploitée au cours d'une année) [Meyer, 2009].
} 
brésilien des statistiques (IBGE), un ralentissement de la progression des effectifs de bovins dans la région alors que les surfaces en pâturages continuent à progresser (Shiki, 1997). Cette tendance se traduit par une baisse du rapport unité animale/surface de pâturage (UA/ha). Cette charge moyenne masque une grande diversité de situations et ne prend pas en compte la diversité des pratiques de gestion des pâturages. Cette charge inférieure au potentiel d'un «bon pâturage» (estimé par l'EMBRAPA-Cerrados à $1 \mathrm{UA} / \mathrm{ha}$ ), et sa baisse témoigneraient donc d'une dégradation. C'est alors, dans ce cas, par rapport à une norme de production attendue à l'échelle de la région qu'est jugée la dégradation.

Des indicateurs non pas de moyens, mais d'état de la pâture sont parfois aussi utilisés : une grille d'évaluation des pâturages (élaborée pour ceux de Brachiaria sp) a été mise au point par l'EMBRAPA-Cerrados (Nascimento, Jr., et al, 1994) et permet de classer les pâturages de «pauvre » à « excellent » sur la base de leur couvert végétal (matière sèche disponible, pourcentage de Brachiaria dans la composition botanique de la pâture, rapport longueur feuille/tige du Brachiaria, hauteur du pâturage) et de signes d'érosion du sol. En outre, Barcellos (1995) identifie quatre étapes du processus de dégradation d'un pâturage : le début du déclin est marquépar la diminution de la production fourragère (en quantité et qualité) ; il est suivi par l'augmentation des surfaces de sol couvertes par les espèces fourragères; puis par l'invasion par des espèces à feuilles larges ; enfin, dans une phase de dégradation avancée, on note une invasion du pâturage par les graminées natives et une érosion avancée du sol. Pour Barcellos (ibid.), les modes actuels de gestion des pâturages par les éleveurs conduisent inéluctablement à un début de déclin au bout de quatre ans après l'implantation du pâturage et, au bout de huit ans, à une dégradation avancée.

S'il existe bien entendu des variations autour de ce discours chez les chercheurs comme chez les techniciens, il n'en reste pas moins que ces indicateurs (et surtout l'évaluation de Barcellos, selon laquelle $80 \%$ des pâturages sont dégradés, évaluation basée sur le premier indicateur de moyen mesurantl'écart entre les pratiques et les recommandations) ont été amplement repris pour justifier de nombreux projets de recherche et de développement et donner ainsi corps au problème de dégradation dans les Cerrados. Le concept de dégradation tel qu'il apparaît à travers la définition du problème permet avant tout de condamner tout ce qui fait obstacle à l'intensification et de dévaloriser l'écart entre les recommandations élaborées par les chercheurs et les pratiques des agriculteurs. Ainsi, « la description scientifique la plus strictement constatative est toujours exposée à fonctionner comme prescription capable de contribuer à sa propre vérification en exerçant un effet de théorie propre à favoriser l'avènement de ce qu'elle annonce » (Bourdieu, 1982, p. 158).
En ce sens, nous pouvons dire que le discours scientifique est en partie performatif : il tire sa vérité de l'autorité de celui qui l'énonce, car, en même temps qu'il énonce l'existence d'une dégradation, il la définit (dégrader, c'est s'éloigner des recommandations des techniciens, c'est refuser d'être moderne) et lui permet ainsi d'exister.

\section{Le lieu de l'action technique : l'agriculture familiale de la commune de Silvânia}

Dans la commune de Silvânia, on trouve, comme dans l'ensemble de la région, de grandes exploitations d'élevage bovin, de grandes exploitations agricoles mécanisées produisant du soja, en rotation avec du maïs, sur plusieurs centaines d'hectares et une majorité de petites exploitations familiales (Encadré). C'est à ces dernières que nous nous intéressons ici.

\section{Encadré. Les exploitations agricoles de l'État du Goiás dans la région des Cerrados}

À la fin des années 1990, on trouve dans la région du Goiás trois types d'exploitations :

- des exploitations agricoles mécanisées de quelques centaines d'hectares, consacrées à la production intensive de soja. Elles représentent le fer de lance du projet de colonisation et de modernisation agricole de la région instauré par le gouvernement dans les années 1960 ;

- de grandes exploitations d'élevage extensif de plusieurs centaines, voire milliers, d'hectares, avec des troupeaux de 300 à 4000 bovins destinés à la production de viande. Elles sont l'héritage du processus de colonisation qui a débuté au XIX ${ }^{\mathrm{e}}$ siècle. Elles sont également appelées à contribuer au projet de développement agricole à travers une augmentation de la charge animale sur des pâturages cultivés ;

- enfin, des exploitations familiales. Définies comme un sous-produit de l'agriculture latifundiste (Bonnal et Zoby, 1994), elles sont localisées dans les interstices géographiques et économiques de cette dernière. Bien que représentant près de $70 \%$ des exploitations de la commune de Silvânia (exploitations de moins de 100 hectares), elles ne couvrent cependant que $17 \%$ des surfaces agricoles. Ces exploitations sont traditionnellement diversifiées : élevage bovin à vocation mixte (lait et viande), quelques porcs et volailles, culture de riz, manioc, maïs, haricot... Les programmes d'appui à la modernisation de ces exploitations, mis en œuvre à la fin des années 1980, vont favoriser une spécialisation de leurs activités à travers la diffusion d'un paquet technique orienté vers l'intensification laitière. 
Les exploitations familiales de la région sont le produit de l'installation ancienne de mineurs, esclaves ou métayers, de la fragmentation progressive des grands domaines coloniaux, puis de l'arrivée dans les années 1960 de petits agriculteurs migrants originaires du Sud et du Nord-Est du pays. L'encadrement technique qui leur est proposé dans les années 1950 est de type « assistanat humaniste » (Rodrigues, 1997) : il est le fait de techniciens en économie domestique et vise à améliorer les conditions de vie des populations rurales. Ces exploitations seront tenues à l'écart des activités de vulgarisation de type "diffusionnisme productiviste", porteuses du projet de modernisation agricole des années 1960 et 1970 dans les Cerrados. Ce n'est qu'à partir de 1985, dans un contexte de démocratisation, que les pouvoirs publics vont mettre en place un programme national d'appui spécifique à l'agriculture familiale (le PRONAF) - avec attribution de crédits spécifiques -, qui reconnaît la contribution de ces exploitations au développement économique de la région. La modernisation touche alors les petites exploitations (Bainville et al., 2005). À Silvânia, comme dans l'ensemble de l'État du Goiás, on constate ainsi une importante augmentation des surfaces en pâturages artificiels, laquelle témoigne d'un processus d'intensification et de spécialisation laitière d'exploitations auparavant diversifiées. Ce processus repose sur une offre technique uniforme (amélioration génétique, amélioration de l'alimentation des animaux en saison sèche, mise en place de pâturages à rotation rapide), diffusée par un encadrement agricole soucieux de sensibiliser les agriculteurs à la dégradation de leurs pâturages.

Dans l'ensemble, les éleveurs se sont largement approprié ce paquet technique, mais leurs pratiques restent cependant en grande partie éloignées des recommandations des techniciens, notamment en ce qui concerne l'implantation et l'entretien des pâturages. Par exemple, $70 \%$ des pâturages des exploitations familiales de Silvânia implantés il y a plus de dix ans n'ont pas fait l'objet des procédures d'entretien recommandées (ni travail du sol, ni resemis, ni fertilisation, d'après notre enquête). Ce qui peut être interprété, selon la définition « experte » de la dégradation explicitée plus haut, par « $70 \%$ des pâturages sont dégradés ", puisque cette définition établit un lien direct entre la mise en œuvre de certaines pratiques et la dégradation, sans que cela soit validé par une évaluation de l'état des ressources.

Pour les techniciens, « les pâturages [des exploitations familiales de la commune] ne sont pas bons ", comme le dit l'un d'entre eux, car ils n'ont pas été implantés ni entretenus correctement. Cependant, ils estiment que l'état des pâturages s'améliore: ces derniers seraient meilleurs qu'auparavant, car le travail de «sensibilisation » des éleveurs porterait ses fruits. En contraste avec l'évaluation des techniciens, et selon la même enquête, les éleveurs sont globalement satisfaits, puisqu'ils qualifient la majorité de leurs pâturages de bons à moyens. Par ailleurs, on a assisté au cours des douze dernières années à une augmentation régulière de la productivité laitière des surfaces consacrées à l'élevage dans l'ensemble des exploitations familiales, celle-ci étant passée en moyenne de 100 l/ha/an en 1986 à 600 l/ha/an en 1998 (d'après la base de données du réseau de fermes de référence constituée par l'EMBRAPA et le Cirad : cf. Bonnal et al., 1993).

\section{La construction de la pensée technique}

Comment analyser cet écart entre les pratiques des agriculteurs et les recommandations des techniciens et entre les jugements que les uns et les autres portent sur les pâturages? L'analyse du discours des éleveurs selon la méthodologie proposée par Darré (1985) nous permet de distinguer localement deux façons de voir les choses : l'ancienne et la nouvelle. Les éléments qualifiés de « nouveaux » ont pour caractéristiques, d'une part, de n'être pas partagés par tous les agriculteurs de notre échantillon et, d'autre part, d'être associés par ceux qui les évoquent à la période actuelle (aujourd'hui, en opposition à avant ou autrefois), à la technologie et à la modernité. Nous parlerons par la suite d'un "système sociotechnique traditionnel » et d'un « système sociotechnique associé au paquet technique ». Il ne s'agit pas, par là, d'opposer agriculteurs traditionnels et modernes. C'est bien au sein du même groupe coactif dans la production de normes que les anciennes façons de voir les choses se transforment, certains individus y étant certes plus actifs que d'autres, plus influents que d'autres. Mais, au sein de ce groupe, il y a convergence sur la nouvelle façon de voir les choses, même si celle-ci coexiste avec l'ancienne et que toutes les formes intermédiaires sont probablement présentes.

Nous examinons successivement comment les agriculteurs définissent et classent les pâturages, comment ces derniers s'insèrent dans le système d'élevage et comment les agriculteurs les jugent.

\section{Pâturages naturels et pâturages artificiels}

Pour les éleveurs de la commune, le mot « pâturage » (pasto, pastagem) est souvent substitué par le mot « herbe » (capim) et il est le sujet de verbes qui se réfèrent au couvert végétal : le pâturage est sec, le pâturage pousse bien...

La conséquence directe est que les pâturages sont classés et évalués selon ce couvert végétal. On distingue traditionnellement les pâturages naturels des pâturages formados (ou artificiels, cultivés, «formés ») - implantation et semis de Brachiaria, Andropogon, Pennisetum. Aux pâturages naturels, c'est-à-dire une savane plus ou moins arbustive, tous associent un ensemble de traits négatifs tels que «sale», «brut». Aux pâturages artificiels, sont 


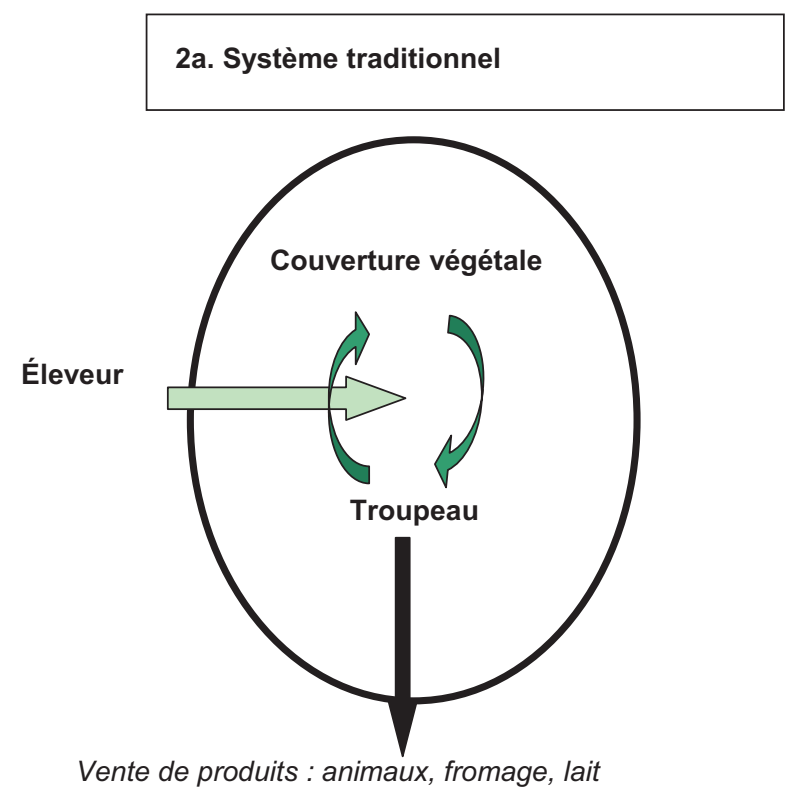

2b. Système associé au paquet technique

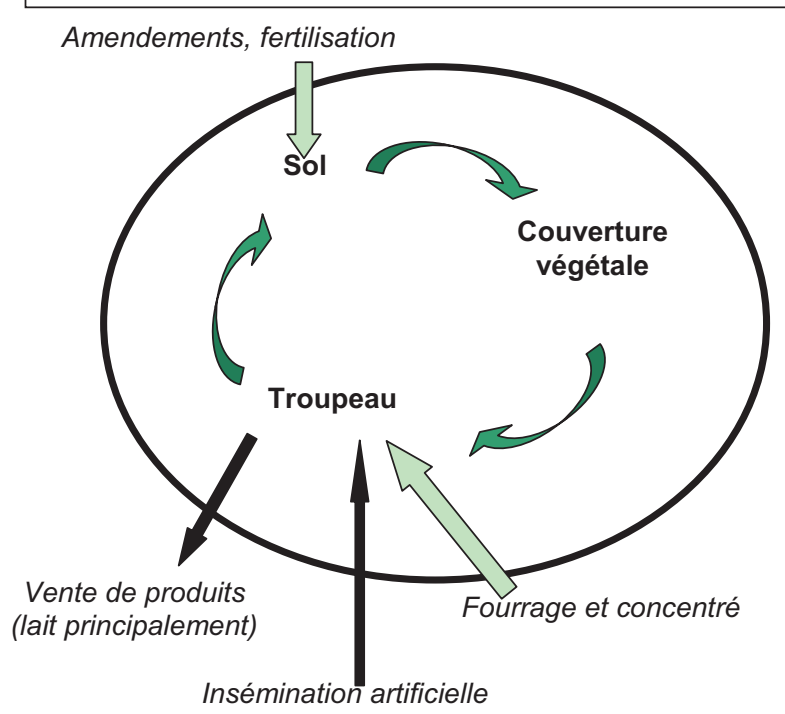

Le pâturage : une force $\quad \Rightarrow$ un élément Une couverture végétale $\Rightarrow$ un sol

Fig. 2. Les systèmes d'élevage vus par les éleveurs. Le système sociotechnique traditionnel et le système sociotechnique associé au paquet technique.

au contraire associés des traits positifs tels que « propre » et « beau ».

\section{Les aires piquetées et les aires non piquetées}

On constate cependant dans le discours des éleveurs que les catégories «naturel » et « artificiel » sont en voie de redéfinition et que leurs contours sont parfois flous. On note, en parallèle, l'apparition chez certains éleveurs d'un nouveau mode de classification des pâturages : les surfaces piquetées ${ }^{9}$ et les surfaces non piquetées. Les premières correspondent aux pâturages à rotation rapide (où les animaux changent de parcelle quotidiennement) ; les secondes, à toutes les autres surfaces.

Les pâturages piquetés sont associés à la modernité (c'est le nouveau message des techniciens), aux coûts (il faut fertiliser deux à trois fois par an), au travail (il faut déplacer quotidiennement les animaux et les clôtures électrifiées) et au lait (à cause de leur coût notamment, ces pâturages sont réservés au troupeau en production).

Parallèlement à cette redéfinition des classes de pâturages apparaît une nouvelle conception du système d'élevage lui-même.

9 C'est ainsi (areas piquetadas) que les éleveurs désignent les surfaces exploitées en pâturage rationné à l'aide de piquets et clôtures mobiles (en gestion tournante).

\section{Le système traditionnel}

Traditionnellement, le système d'élevage est perçu comme une combinaison de forces à gérer : d'un coté, la force de la terre et de l'herbe ; de l'autre, celle de la vache. L'agriculteur parle ainsi de sa vache qui « force l'herbe » ou de l'herbe qui « perd de la force ». Le rôle de l'éleveur est de gérer l'équilibre de ces forces par des actions sur le couvert végétal (nettoyage) et par la gestion de la charge (mise en réserve notamment) [Fig. 2a].

Chez les éleveurs qui partagent cette conception, le système est peu artificialisé (pas de correction des sols, grande proportion de pâturages naturels à l'intérieur de l'exploitation, animaux de race rustique). Leur tâche est, pour eux, de gérer un potentiel naturel en combinant des forces au sein d'un système complexe d'éléments en interactions. Dans ce système, il s'agit en particulier d'accepter les limites du potentiel naturel de ces éléments et de $\mathrm{s}^{\prime} \mathrm{y}$ adapter.

\section{Le système associé au paquet technique}

Chez les éleveurs ayant adopté le paquet technique proposé par les techniciens, on note que le système d'élevage (Fig. 2b) s'apparente davantage à une chaîne de 

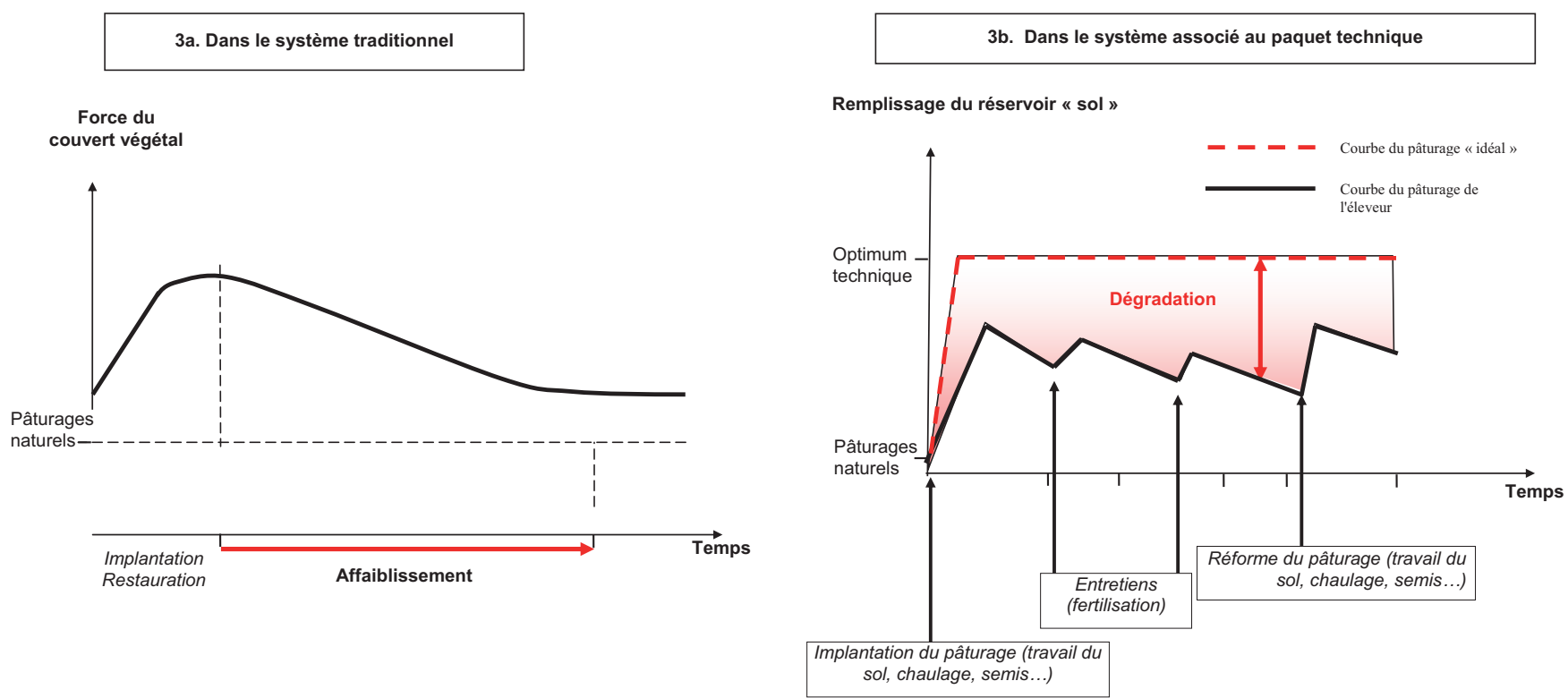

Fig. 3. Les représentations de la dégradation des pâturages par les éleveurs.

causalités objectivées, entre des actions et des éléments identifiés reliés entre eux, à l'image d'un système de vases communicants : le producteur apporte au sol les éléments nécessaires à la croissance du pâturage, lequel à son tour apporte au troupeau l'alimentation nécessaire à la production de lait, la vente de ce dernier permettant l'achat $\mathrm{d}$ 'engrais et le renouvellement du système. On note ici, au passage, l'apparition d'une nouvelle composante : le sol, quasiment absent du système traditionnel précédemment décrit.

Ce qui met en cohérence les éléments du système associé au paquet technique est cet enchaînement en vases communicants ; la performance du système est liée à la capacité d'un des éléments à transmettre l'apport réalisé à l'élément suivant. Cette capacité est le résultat d'un travail de construction, car chaque élément du système d'élevage est ici construit par l'éleveur. Ainsi :

- les sols sont corrigés, fertilisés. L'agriculteur « construit la fertilité du sol », selon l'expression utilisée par les techniciens ;

- les pâturages sont pour la plupart artificiels ;

- le troupeau est amélioré génétiquement (race Holstein).

Le rôle de l'éleveur n'est plus de gérer un potentiel naturel et des équilibres entre des forces, comme dans le système traditionnel précédent. Il lui faut construire ce potentiel et l'entretenir en faisant en sorte que soit apporté au système au moins autant que ce qui en a été retiré.

Compte tenu des caractéristiques des "piquets ", que nous avons présentées, ils ne peuvent s'insérer dans le système traditionnel. En effet, ils nécessitent des investissements (dépense de fertilisation, travail de déplacement...) dont l'éleveur attend un retour immédiat. Or, dans le système traditionnel, les éléments ne rendent pas nécessairement ce qu'on leur accorde, leur réponse dépend de leur potentiel naturel ${ }^{10}$. L'adoption des piquets et des pratiques de fertilisation qui y sont liées, mais également des nouvelles races bovines (Holstein), ne pouvait se faire que dans le cadre d'une reconstruction de la représentation des systèmes d'élevage. Les piquets et les nouvelles races bovines trouvent leur place dans ce nouveau système où les éléments sont davantage maîtrisés par l'éleveur.

\section{La formulation d'un concept local de dégradation}

\section{De l'affaiblissement des pâturages...}

La plupart des agriculteurs n'utilisent pas le mot dégradation (degradaçao), voire ne le comprennent même pas. Mais ils parlent de pâturages faibles ou qui s'affaiblissent, et cet affaiblissement est localisé au niveau du couvert végétal (Fig. 3a) : «l'herbe est faible », « le pâturage s'affaiblit ». Cette diminution de la force est essentiellement associée à l'âge du pâturage (défini ainsi à partir de sa date d'implantation). L'éleveur considère que c'est un phénomène normal, qu'il ne peut empêcher mais seulement ralentir par certaines pratiques (notamment en

10 Ainsi, une « mauvaise vache » (c'est-à-dire bien souvent, dans le discours des éleveurs, une vache de race locale) ne rend pas nécessairement ce qu'on lui donne : A Alimenter une mauvaise vache est comme alimenter une bonne. Ça coûte la même chose. Sauf qu'une bonne vache rapporte un bénéfice», explique un éleveur. 
jouant sur la charge et les dates de pâture). C'est la nature qui reprend le dessus, les espèces spontanées se multiplient, diminuant la biomasse des espèces considérées comme fourragères ; $c^{\prime}$ est le retour de l'artificiel au naturel. Le pâturage est alors la première étape d'une jachère arborée. Etl'on peut vérifier dans le discours que les caractéristiques d'un " mauvais " pâturage sont en tout point comparables à celles du pâturage naturel : sale, sec. Le pâturage naturel sert donc de référence à la qualification des pâturages.

\section{... à leur dégradation}

$\mathrm{Si}$, traditionnellement, le pâturage est jugé par son couvert végétal et en fonction de son âge, pour les éleveurs qui ont adopté le paquet technique un autre niveau de jugement intervient : le sol (Fig. 3b). Ces éleveurs utilisent spontanément le mot dégradation, qu'ils assimilent à une baisse de fertilité des sols : « La dégradation fut telle, c'est-à-dire la fertilité a chuté... " Dans ce système de vases communicants, le pâturage dégradé est celui « qui ne répond plus ». La dégradation vient du fait qu' on retire plus qu'on a apporté : le réservoir sol s'est vidé. Le mot dégradation a été introduit par les techniciens en même temps que les analyses et les conseils personnalisés de fertilisation, révélant ainsi aux éleveurs une nouvelle dimension deleurs pâturages : le sol. La dégradation n'est pas une évaluation par rapport à un potentiel naturel, qui n'existe pas ou quasiment pas dans la vision techniciste, ni même par rapport à un potentiel passé, même construit par l'éleveur. Car, lorsque ces éleveurs parlent de dégradation, ils font davantage référence à un potentiel futur qu'il leur semble possible d'atteindre s'ils appliquent les nouvelles technologies, notamment en corrigeant les déficiences des sols. La référence pour l'évaluation des pâturages est ici la norme du technicien. Ainsi, si ces éleveurs «modernes» peuvent juger leurs pâturages dégradés, ils ne les jugent généralement pas en voie de dégradation. Au contraire, ils estiment que leurs pâturages s'améliorent, car, même s'ils restent en dessous de la norme, ils s'en rapprochent. Ces éleveurs parlent ainsi avec une certaine fierté de leurs pâturages, qu'ils qualifient pourtant de dégradés, car ils indiquent par là le niveau élevé de leurs exigences, en relation avec les progrès techniques qu'ils connaissent et qu'ils sont prêts à appliquer.

\section{Les difficultés du changement de normes pour les éleveurs}

Adopter le paquet technique de l'intensification laitière exige donc de la part des éleveurs un changement également dans la façon de penser leurs pâturages et de les évaluer. Ce changement de normes ne se fait pas sans difficultés. En effet, le discours du technicien témoigne d'un mot d'ordre omniprésent de stabilité : stabilité des effectifs animaux sur l'exploitation; stabilité de la production laitière sur l'année; stabilité de l'état des pâturages. Un état stable du pâturage suppose de la part du producteur des interventions avant même que le couvert végétal ou les animaux aient donné le moindre signe de déclin. La dégradation, c'est l'état constaté quand on s'éloigne de la courbe du pâturage «idéal » (Fig. 3b) : elle doit être évitée par une fertilisation d'entretien régulière (par apports de superphosphate, urée, sulfate d'ammonium), sur des sols corrigés (par apports de calcaire) en fonction des résultats d'analyses des sols.

\section{La perte de référence temporelle et spatiale}

Dans le système associé au paquet technique, l'affaiblissement fait place à la dégradation. Ce changement s'accompagne d'une perte de références temporelle et spatiale. En effet, contrairement à l'affaiblissement des pâturages (Fig. 3a), la dégradation (Fig. 3b) est un état et non pas un processus. Le facteur temps a disparu. Ainsi, la connaissance qu'a le producteur de l'histoire de la parcelle et de sonévolution nelui est pas utileici. D'autre part, les pâturages appartiennent à un système fonctionnel de parcelles dont l'éleveur n'attend pas les mêmes qualités de chacune d'elles. C'est d'ailleurs sur cette variabilité que repose sa capacité à exploiter la diversité des conditions du milieu dans son exploitation (Hubert, 1994). Or, la dégradation telle que définie par le discours scientifique s'apprécie à l'échelle de la parcelle, sans connaissance nécessaire de l'ensemble du système fonctionnel de parcelles, dans lequel celles dites « sales », partiellement envahies de buissons, jouent un rôle de réserve sur pied pour les périodes sèches. La connaissance qu'a le producteur de son exploitation, du potentiel naturel des différentes zones, des complémentarités possibles entre ces zones, n'est pas non plus mobilisable dans l'élaboration du diagnostic de dégradation.

Juger de la dégradation selon les critères du discours scientifique, c'est alors pour le producteur faire abstraction de la connaissance de l'histoire et de la variabilité spatiale qu'il a de son exploitation pour se mettre au même niveau d'ignorance que le technicien face à celle-ci.

\section{Gérer la dégradation : piloter les yeux fermés}

Si le discours sur la dégradation se construit sur une perte de références temporelle et spatiale, il se construit aussi, comme nous l'avons vu précédemment, sur l'introduction d'une nouvelle référence : le sol. S'approcher de l'optimum de productivité des pâturages et de la courbe plate du technicien, $\mathrm{c}^{\prime}$ est, selon le concept scientifique, corriger, fertiliser ses sols et abandonner le repère 
végétation (qui traditionnellement reflète l'aptitude culturale d'une terre: Affholder et al., 2003), trompeur car reflétant imparfaitement et avec retard l'état réel du sol - « L'herbe trompe le producteur », dit un technicien. Sans repère, il est demandé à l'éleveur de piloter son système les yeux fermés. Le dégradé devient alors pour lui le domaine de l'inconnu et de l'inaccessible. Il a nécessairement recours à des indicateurs $(\mathrm{pH}$, taux de phosphore, de calcaire...) produits à l'extérieur de ses propres savoirs, et par là même les déniant : l'agriculteur doit recourir à des analyses de sol pour se situer par rapport à une norme construite à l'extérieur de l'exploitation et du groupe d'agriculteurs.

\section{La dégradation par changement de fonction}

Nous avons posé en première partie que les discours sur la dégradation des ressources naturelles tiraient une grande partie de leur sens de la fonction attribuée à ces ressources et que la diversité des sens donnés au mot dégradation était liée à la diversité des fonctions attribuées à ces ressources.

Dans le cas des éleveurs de notre échantillon, on constate que l'adoption, même partielle, du concept « technicien » de dégradation n'est pas liée à la reconnaissance, ou à la prise de conscience, de l'existence d'un changement d'état des parcelles, puisque, globalement, les éleveurs pensent que leurs pâturages sont satisfaisants et que leur état s'est amélioré. C'est l'adoption de l'objectif d'intensification qui, en modifiant la fonction des pâturages, rend l'adoption du concept de dégradation possible. En particulier, les exigences relatives aux pâturages destinés à l'alimentation des vaches en production ont augmenté. Cela explique que le discours des agriculteurs qui parlent de dégradation porte quasi exclusivement sur les « pâturages à lait » et pourquoi c'est sur ces pâturages que leurs jugements sont le plus sévères, en relation avec un niveau d'exigence élevé. Ainsi, la dégradation apparaît bien par changement de fonction de la ressource et non pas par son changement d'état.

\section{Conclusion}

Le paquet technique transmis aux éleveurs de Silvânia témoigne d'une vision de l'innovation "clef en main », laquelle repose sur l'adoption de l'ensemble de ce paquet par les éleveurs et néglige leurs activités de construction et de conception.

Mais surtout, l'exemple étudié est révélateur de l'approche que les sciences agronomiques ont des ressources naturelles. Les sciences agronomiques considèrent généralement les ressources naturelles comme un capital, plus ou moins limité et plus ou moins renouvelable. Lorsqu'une des composantes de ce capital est jugée insuffisante pour garantir la pérennité du système utilisateur, il convient donc soit d'en réduire le taux de consommation, soit d'en améliorer le rendement d'exploitation, soit de la substituer par un autre type de ressource. En matière de gestion des espaces pastoraux, cette vision est à l'origine des recommandations habituelles sur la maîtrise des chargements aussi bien que de celles visant à introduire des pâturages plus productifs ou à augmenter la production de biomasse par des apports extérieurs, fertilisants ou autres. C'est ce que Thompson (1997) désigne sous le nom de « resource sufficiency ».

Cette approche reposant sur le traitement hiérarchisé des facteurs limitants n'est pas toujours pleinement opérante. C'est le cas quand elle s'applique à des systèmes complexes, comme les systèmes pastoraux réputés " extensifs » ou "traditionnels », tels ceux rencontrés à Silvânia. La diversité des ressources mobilisées (espaces cultivés, espaces naturels divers), des fonctions de l'élevage : productions (lait, viande, laine, cuir), travail (traction, transports), transferts de fertilité, des services (conservation de la biodiversité et des paysages), impose de considérer des interactions dont une approche analytique classique ne peut rendre totalement compte. Travailler sur la durabilité de la gestion des ressources oblige alors à identifier les stratégies envisageables pour ces systèmes qui préservent leur capacité de résilience et d'évitement d'irréversibilités, dans des contextes d'incertitudes fortes liées aux dynamiques de facteurs externes: économiques, sociaux ou climatiques. Cela signifie s'intéresser à leur intégrité fonctionnelle («functional integrity », selon Thompson [1997]). C'est ce que proposent Hubert et Ison (2011) à partir de l'analyse de neuf études de cas sur des situations d'élevage provenant des cinq continents (Kammili et al., 2011).

Parler d'intégrité fonctionnelle renvoie à l'idée que le système s'inscrit de façon dynamique dans un espace de viabilité, au sein duquel la reproduction de ses élémentsclés est effectivement possible sur une longue période de temps. Des crises peuvent cependant apparaître, conséquence plus ou moins immédiate d'une altération critique de la capacité de reproduction d'un ou plusieurs de ces éléments, altération elle-même liée aux pratiques et à leur évolution ou à l'inadaptation des pratiques habituelles à des changements externes des conditions de reproduction de ce ou ces éléments (par exemple, le changement climatique). Dans cette perspective, les ressources ne peuvent donc pas être considérées dans l'absolu, indépendamment des pratiques par lesquelles elles sont mobilisées et donc des valeurs, des références, des normes qui sous-tendent ces pratiques et doivent être prises en compte comme des composantes à part entière de l'agroécosystème.

En imposant, à l'instar des techniciens de Silvânia, un point de vue différent de la vision dynamique et complexe des forces du monde des ressources, en proposant une 
vision constituée d'éléments stabilisés, on a conçu un raisonnement dominant à l'égard des ressources, comme si celles-ci constituaient un capital - un stock - donné une fois pour toutes et qu'elles étaient classables en « renouvelables », « non renouvelables », « critiques », etc. Mais si on regarde les ressources comme le produit émergent des interactions entre une société et des éléments de son environnement, comme l'orientation, au profit des groupes humains, des fonctionnalités des écosystèmes au sein desquels ils vivent, alors on ouvre de nouvelles perspectives cognitives et praxéologiques!

Ainsi, penser en termes d'intégrité fonctionnelle la question de la gestion durable des ressources naturelles implique des recherches qui s'attachent à une meilleure compréhension des facteurs critiques, des composantes d'un système et des interactions systémiques entre cellesci qui conditionnent sa capacité à se reproduire et à s'adapter. L'écologie, la modélisation des écosystèmes, l'analyse systémique, mais aussi les sciences agronomiques et les sciences humaines et économiques sont donc essentielles dans cette approche, impérativement interdisciplinaire. De plus, la complexité des problèmes et des enjeux invite à une démarche qui confronte et fasse dialoguer les connaissances et les méthodes scientifiques «académiques» avec les savoirs empiriques locaux. Ainsi, d'après Scoones et al. (2007), c'est la combinaison $d^{\prime}$ une approche constructiviste et d'une approche des ressources non focalisée sur l'équilibre qui peut ouvrir la voie à une science de la durabilité.

\section{Remerciements}

Nous remercions les responsables du projet Silvânia (1985-1998) : Philippe Bonnal, Jose Luiz Fernandes Zoby, Marcelo Leite Gastal et Jose da Silva Netto Madeira. Nous remercions également François Affholder (Cirad), ainsi que Laura Duarte et Maria Stela Grossi Porto du département de sociologie de l'Université de Brasilia (UnB).

\section{Références}

Affholder, F., Scopel, E., Neto, J.M., Capillon, A., 2003. Diagnosis of the productivity gap using a crop model. Methodology and case study of small-scale maize production in central Brazil, Agronomie, 23, 4, 305-325.

Bainville, S., Affholder, F., Figuié, M., Madeira, J.S.N., 2005. Les transformations de l'agriculture familiale de la commune de Silvânia: une petite révolution agricole dans les Cerrados brésiliens, Cahiers Agricultures, 14, 1, 103-110.

Barcellos, A de O., 1995. Recuperaçao de pastagens degradadas, Nota da EMBRAPA-CPAC, CRI 3795.

Barcellos, A. de O., 1996. Sistema extensivos e semi-extensivos de produção: pecuária bovina de corte no Cerrado, in Pereira R.C., Nasser L.C.B. (Eds), Anais do $8^{\circ}$ Simpósio sobre $o$ Cerrado, Planaltina, EMBRAPA-CPAC.
Bertran, P., 1988. Uma introduçao a historia economica do CentroOeste do Brasil, Brasilia, Codeplan / Goiânia, UCG.

Bertran, P., 1994. Historia da terra et do homen do Planalto Central: Eco historia do Distrito Federal, do indigena ao colonizador, Brasilia, Solo.

Bonnal, P., Xavier, J.H.V., dos Santos, N.A., de Souza, G.L.C., Zoby, J.L.F., Gastal, M.L., Pereira, E.A., Paniago, Jr., E., de Souza, J.B., 1993. O papel da rede de fazendas de referência no enfoque de Pesquisa/Desenvolvimento. Projeto Silvânia, Planaltina-DF, EMBRAPA-CPAC/CIRAD-SAR.

Bonnal, P., Zoby, J.L.F., 1994. Pesquisa-desenvolvimento e sustentabilidade nos Cerrados. caso do projeto Silvânia. Seminario PROCITROPICOS: “Diversidad y dinámica de la unidades y sistemas de producción: condiciones y parámetros socio-económicos de la sostenibilidad", Brasilia

Bourdieu, P., 1982. Ce que parler veut dire : l'économie des échanges linguistiques, Paris, Fayard.

Bourdieu, P., Wacquant, L., 1992. Réponse, Paris, Le Seuil.

Callon, M., Rip, A., 1992. Humains, non-humains : morale d'une coexistence, in Theys, J., Kalaora, B. (Eds), La Terre outragée : les experts sont formels !, Paris, Autrement, 140-156.

Darré, J.-P., 1985. La Parole et la technique : l'univers de pensée des éleveurs du Ternois, Paris, L'Harmattan.

Darré, J.-P., 1999. La Production de connaissance pour l'action: arguments contre le racisme de l'intelligence, Paris, Éditions de la Maison des sciences de l'homme / INRA.

Duarte, L.M.G., Braga, M.L de S., Bezerra, C. da S. (Eds), 1998. Tristes Cerrados: Sociedade e biodiversidade, Brasília, Paralelo 15.

Figuié, M., 2001. La Construction sociale d'un savoir sur la dégradation des ressources naturelles : le cas des pâturages dans les exploitations agricoles de la commune de Silvânia au Brésil. Thèse de doctorat, INA P-G, Paris.

Forum brasileiro de ONGs e movimentos sociais, 1997. Brasil século XXI: Os caminhos da sustentabilidade. Cinco anos depois de Rio 92, Rio de Janeiro, FASE.

Geels, F.W., 2004. From sectoral systems of innovation to sociotechnical systems: Insights about dynamics and change from sociology and institutional theory, Research Policy, 33, 6-7, 897-920.

Hatchuel, A., 2005. Towards an epistemology of collective action: Management research as a responsive and actionable discipline, European Management Review, 2, 36-47.

Hubert, B., 1994. Pastoralisme et territoire : modélisation des pratiques d'utilisation, Cahiers Agricultures, 3, 9-22.

Hubert, B., Ison, R., 2011. Institutionalising understanding: From resource sufficiency to functional integrity, in Kammili, T., Hubert, B., Tourrand, J.-F. (Eds), A Paradigm Shift in Livestock Management: From Resource Sufficiency to Functional Integrity, Lirac (France), Cardère éditeur, 11-16.

Kammili, T., Hubert, B., Tourrand, J.-F., 2011. A Paradigm Shift in Livestock Management: From Resource Sufficiency to Functional Integrity, Lirac (France), Cardère éditeur.

INESC (Instituto de estudos sócio-econômicos), 1994. Meio Ambiente, Fome e Miseria: Um caso do Cerrado Mineiro. Subsidio 20, Brasília, INESC.

Larrère, C., Larrère, R. (Eds), 1997. La Crise environnementale, Paris, INRA.

Latour, B., 1995. Le Métier de chercheur: regard d'un anthropologue, Paris, INRA. 
Macedo, J., 1995. Os Cerrados brasileiros: Alternativas para a produção de alimentos no limiar do século XXI, Revista de Política Agrícola, 2, 11-18.

Meyer, C. (Ed.), 2009. Dictionnaire des sciences animales. Montpellier, France, http:/ / dico-sciences-animales.cirad.fr/ (consulté le 23 juin 2009).

Moscovici, S., 1961. La Psychanalyse, son image et son public, Paris, PUF.

Nascimento Junior, D. do, Savio Queiroz, D., Santos, M.V.F., 1994. Degradaçao das pastagens e criterios para avaliaçao, in Peixoto, A.M., Moura, J.C., Faria, V.P. (Eds), Anais do $11^{\circ}$ Simposio sobre Manejo de Pastagem, Piracicaba, FEALQ, 107151.

Pinto, M.N. (Ed.), 1993. Cerrado: caracterização, occupação e perspectivas, Brasilia, Ed. Universidade de Brasilia.
Rodrigues, C.M., 1997. Conceito de seletividade de politicas publicas e sua aplicaçao no contexto da politica de extensao rural no Brasil, Cadernos de Cienciae Tecnologia, 8, 1-3, 113-154.

Scoones, I., Leach, M., Smith, A., Stagl, S., Stirling, A., Thompson, J., 2007. Dynamic Systems and the Challenge of Sustainability. STEPS Working Paper, 1, Brighton, STEPS Centre.

Shiki, S., 1997. Sistema agroalimentar no Cerrado brasileiro: caminhando para os caos?, in Shiki, S., Silva, J.G., Ortega, A.C. (Eds), Agricultura, meio ambiente e sustentabilidade do Cerrado brasileiro, Uberlândia, EMBRAPA/CNPAMA, 135167.

Thompson, P., 1997. The varieties of sustainability in livestock farming, in Sørensen, J.T. (Ed.), Livestock Farming Systems: More than Food Production, Wageningen, Wageningen Press, $5-15$.

Reçu le $1^{\text {er }}$ septembre 2010. Accepté le 6 octobre 2011. 